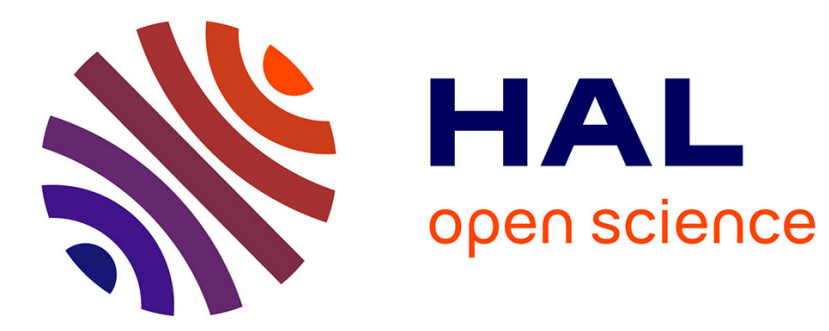

\title{
Multidimensional analytic signal with application on graphs
}

Mikhail Tsitsvero, Pierre Borgnat, Paulo Gonçalves

\section{To cite this version:}

Mikhail Tsitsvero, Pierre Borgnat, Paulo Gonçalves. Multidimensional analytic signal with application on graphs. SSP 2018 - IEEE Workshop on Statistical Signal Processing, IEEE, Jun 2018, Freiburg, Germany. pp.1-5. hal-01878753

\section{HAL Id: hal-01878753 \\ https://hal.inria.fr/hal-01878753}

Submitted on 21 Sep 2018

HAL is a multi-disciplinary open access archive for the deposit and dissemination of scientific research documents, whether they are published or not. The documents may come from teaching and research institutions in France or abroad, or from public or private research centers.
L'archive ouverte pluridisciplinaire HAL, est destinée au dépôt et à la diffusion de documents scientifiques de niveau recherche, publiés ou non, émanant des établissements d'enseignement et de recherche français ou étrangers, des laboratoires publics ou privés. 


\title{
MULTIDIMENSIONAL ANALYTIC SIGNAL WITH APPLICATION ON GRAPHS
}

\author{
Mikhail Tsitsvero $^{(1)}$, Pierre Borgnat ${ }^{(2)}$, Paulo Gonçalves ${ }^{(1)}$ \\ (1) Univ Lyon, Inria, CNRS, ENS de Lyon, UCB Lyon 1, LIP UMR 5668, F-69342, Lyon, France \\ (2) Univ Lyon, ENS de Lyon, UCB Lyon 1, CNRS, Laboratoire de Physique, F-69342 Lyon, France
}

\begin{abstract}
In this work we provide an extension to analytic signal method for multidimensional signals. First, expressions for separate phase-shifted components are given. Second, we show that phase-shifted components could be obtained from hypercomplex Fourier transform in the algebra of commutative hypercomplex numbers. Finally we apply multidimensional analytic signal method to signals defined on graphs.
\end{abstract}

Index Terms - Analytic signal, hypercomplex and Clifford Fourier transforms, Hilbert transform, analytic signal on graph, diffusion maps

\section{INTRODUCTION}

Analytic signal method [1], [2] has found many applications in science and engineering. Appropriate generalizations of analytic signals to two or more dimensions and its connection with complex and hypercomplex analysis were studied in the recent decades. This was motivated by the development of signal processing methods for image analysis $[3,4,5,6]$ and analysis of multivariate signals $[7,8,9,10,11,12,13,14,15]$.

The main issue of the present work is how to compute the phase-shifted versions of a real-valued function in many dimensions, so that one can compute a multidimensional analytic signal satisfying the classical properties of 1-D analytic signals, especially offering the possibility to estimate the envelope of an oscillating signal. We show here that the commutative Scheffers hypercomplex algebra is convenient to compute a multidimensional analytic signal, using the corresponding hypercomplex Fourier transform.

Presented approach generalizes the standard results known for 1-D signals from four different and complementary perspectives: 1) by using Fourier integral formula; 2) by projection and reconstruction on phase-shifted harmonics; 3) using multidimensional Hilbert transform, and 4) by considering the positive frequency restriction of hypercomplex Fourier transform.

Second, we propose a method to construct analytic signal on graph. The motivation comes from graph-based machine

This work was supported by Labex MILyon and ANR-14-CE27-0001 GRAPHSIP grant. learning methods [16, 17], graph signal processing [18] and graph characterization methods $[19,20]$.

In the context of graph signal processing, analytic signals on graphs were considered in [21,22]; however their treatment of Hilbert transform for graphs is essentially one dimensional and relies on the asymmetry of the diffusion operator on the graph, like the adjacency matrix, therefore it does not extend to undirected graphs.

In Section 2, we introduce the concept and properties of multidimensional analytic signal and explain how to calculate it using Scheffers hypercomplex algebra. The proofs of the theorems are omitted and are detailed in [23]. Then, in Section 3 , we provide numerical illustrations for graph analytic signals, which relies on the mapping of the original signal to a multidimensional signal using diffusion maps [24].

\section{ANALYTIC SIGNAL IN MANY DIMENSIONS}

For convenience we introduce the following notation. For a given binary $\{0,1\}$-string of length $d, \boldsymbol{j} \in\{0,1\}^{d}$, we define the functions $\alpha_{j}: \mathbb{R}^{d} \times \mathbb{R}^{d} \rightarrow \mathbb{R}$ by

$$
\alpha_{\boldsymbol{j}}(\boldsymbol{x}, \boldsymbol{\omega}):=\prod_{l=1}^{d} \cos \left(\omega_{l} x_{l}-j_{l} \frac{\pi}{2}\right) .
$$

Definition 2.1. The phase-shifted version $f_{\boldsymbol{j}}(\boldsymbol{x})$, in the direction $\boldsymbol{j} \in\{0,1\}^{d}$, of a real-valued function $f(\boldsymbol{x})$ is given by

$$
f_{j}(\boldsymbol{x})=\frac{1}{\pi^{d}} \int_{0}^{\infty} \int_{-\infty}^{\infty} f\left(\boldsymbol{x}^{\prime}\right) \alpha_{j}\left(\boldsymbol{x}-\boldsymbol{x}^{\prime}, \boldsymbol{\omega}\right) \mathrm{d} \boldsymbol{x}^{\prime} \mathrm{d} \boldsymbol{\omega} .
$$

Definition 2.2. We call

- instantaneous amplitude or envelope $a(\boldsymbol{x})$, the following combination of all phase-shifted versions of the signal:

$$
a(\boldsymbol{x}):=\sqrt{\sum_{\boldsymbol{j} \in\{0,1\}^{d}} f_{\boldsymbol{j}}(\boldsymbol{x})^{2}}
$$

- instantaneous phase $\phi_{\boldsymbol{j}}(\boldsymbol{x})$ in the direction $\boldsymbol{j} \in\{0,1\}^{d}$, the angle:

$$
\phi_{\boldsymbol{j}}(\boldsymbol{x}):=\arctan \left(\frac{f_{\boldsymbol{j}}(\boldsymbol{x})}{f(\boldsymbol{x})}\right) .
$$


The quantity $a(\boldsymbol{x})$ represents a norm [25] defined in the Banach space of commutative hypercomplex numbers that we introduce shortly. The instantaneous frequency may be defined naturally [23] by taking partial derivatives for each direction $j \in\{0,1\}^{d}$. The definition of a Hilbert transform in a $d$-dimensional space follows from the definition in 1-D.

Definition 2.3. The Hilbert transform of a function $f: \mathbb{R}^{d} \rightarrow$ $\mathbb{R}$ in the direction $\boldsymbol{j} \in\{0,1\}^{d}$ is defined by

$$
\left(H_{j} f\right)(\boldsymbol{x})=\text { p.v. } \frac{1}{\pi^{|\boldsymbol{j}|}} \int_{\mathbb{R}^{|\boldsymbol{j}|}} \frac{f(\boldsymbol{y})}{(\boldsymbol{x}-\boldsymbol{y})^{\boldsymbol{j}}} \mathrm{d} \boldsymbol{y}^{\boldsymbol{j}},
$$

where $|\boldsymbol{j}|$ gives the number of $1-\mathrm{s}$ in $\boldsymbol{j},(\boldsymbol{x}-\boldsymbol{y})^{\boldsymbol{j}}=\prod_{i=1}^{d}\left(x_{i}-\right.$ $\left.y_{i}\right)^{j_{i}}$ and $\mathrm{d} \boldsymbol{y}^{j}=\mathrm{d} y_{1}^{j_{1}} \ldots \mathrm{d} y_{d}^{j_{d}}$, i.e. integration is performed only over the variables indicated by the vector $j$.

Thus defined, the multidimensional Hilbert transform binds to the phase shift of Def. 2.1; like in the 1-D case, where the Hilbert transform coincides with its quadrature version. It is stated in the following

Theorem 2.4. For a continuous and absolutely integrable $f(\boldsymbol{x})$, we have

$$
f_{\boldsymbol{j}}(\boldsymbol{x})=\left(H_{\boldsymbol{j}} f\right)(\boldsymbol{x}) .
$$

Continuing the parallel with 1-D, it is of interest to express the phase-shifted function $f_{\boldsymbol{j}}(\boldsymbol{x})$ as a combination of the harmonics $\alpha_{\boldsymbol{j}}(\boldsymbol{x}, \boldsymbol{\omega})$ and the corresponding projection coefficients $\alpha^{j}(\boldsymbol{\omega})$. Indeed, as there exist efficient algorithms implementing sine and cosine transforms, it is plausible that knowing the coefficients $\alpha^{j}(\boldsymbol{\omega})$ will ease the numerical calculation of the phase-shifted functions $f_{j}$.

To this end, we define the projection coefficients as

$$
\alpha^{j}(\boldsymbol{\omega}):=\int_{-\infty}^{\infty} f(\boldsymbol{x}) \alpha_{\boldsymbol{j}}(\boldsymbol{x}, \boldsymbol{\omega}) \mathrm{d} \boldsymbol{x},
$$

and introduce the brackets $\langle a, b\rangle_{+}$for two functions $a(\boldsymbol{\omega})=$ $a\left(\omega_{1}, \ldots, \omega_{d}\right)$ and $b(\boldsymbol{x}, \boldsymbol{\omega})=b\left(x_{1}, \ldots, x_{d}, \omega_{1}, \ldots, \omega_{d}\right)$ as following

$$
\langle a, b\rangle_{+}(\boldsymbol{x}):=\frac{1}{\pi^{d}} \int_{\mathbf{0}}^{\infty} a(\boldsymbol{\omega}) b(\boldsymbol{x}, \boldsymbol{\omega}) \mathrm{d} \boldsymbol{\omega} .
$$

Theorem 2.5. For a given function $f: \mathbb{R}^{d} \rightarrow \mathbb{R}$, the phaseshifted version $f_{\boldsymbol{j}}$ in the direction $\boldsymbol{j} \in\{0,1\}^{d}$ reads

$$
f_{\boldsymbol{j}}(\boldsymbol{x})=\sum_{\boldsymbol{i} \in\{0,1\}^{d}}(-1)^{|(\boldsymbol{i} \oplus \boldsymbol{j}) \ominus \boldsymbol{i}|}\left\langle\alpha^{\boldsymbol{i} \oplus \boldsymbol{j}}(\boldsymbol{\omega}), \alpha_{\boldsymbol{i}}(\boldsymbol{x}, \boldsymbol{\omega})\right\rangle_{+}(\boldsymbol{x}),
$$

where $\oplus$ is a binary exclusive OR operation acting elementwise on its arguments and $\ominus$ is defined as following: $1 \ominus 0=1$ and the result is 0 otherwise. To state it simply: we have "- " sign before the term in the sum when the corresponding upper index inside the bracket is 1 and down index is 0 and " + " sign in any other case.
As a brief demonstration to this theorem we provide an example in 1-D.

Example 2.6. In 1-D case, the analytic signal $f_{a}: \mathbb{R} \rightarrow \mathbb{C}$ of a function $f: \mathbb{R} \rightarrow \mathbb{R}$ is given by $f_{a}(x)=f_{0}(x)+i f_{1}(x)$, where $f_{0}(x)=f(x)$ and $f_{1}(x)=(H f)(x)$, its Hilbert transform. Theorem 2.5 in this case reads

$$
\begin{aligned}
& f_{0}(x)=\left\langle\alpha^{0}(\omega), \alpha_{0}(x, \omega)\right\rangle_{+}+\left\langle\alpha^{1}(\omega), \alpha_{1}(x, \omega)\right\rangle_{+} \\
& f_{1}(x)=\left\langle\alpha^{0}(\omega), \alpha_{1}(x, \omega)\right\rangle_{+}-\left\langle\alpha^{1}(\omega), \alpha_{0}(x, \omega)\right\rangle_{+}
\end{aligned}
$$

It follows that component $f_{0}$ is obtained by combination of inverse cosine transform of the forward cosine transform plus inverse sine transform of the forward sine transform. While the component $f_{1}$ is computed by inverse cosine transform of the forward sine transform minus inverse sine transform of the forward cosine transform of the original function $f$.

\subsection{Scheffers algebra-valued Fourier transform}

One corollary property of analytic 1-D signals defined in the algebra of complex numbers, is that their Fourier spectrum has support only over positive frequencies. During the last few decades a number of works were dedicated to the generalization of the Fourier transform, in order to define analytic signal in several dimensions. These generalizations were mainly based on non-commutative algebras like quaternions, Caley-Dickson and Clifford algebras, see e.g. [10, 26, $27,28,11,3,29,12,30,31,32,33,34]$. In this paper we show that a commutative and associative hypercomplex algebra yields a nice approach to define multidimensional analytic signal. Moreover in [23] we demonstrate that one cannot obtain the phase-shifted components $f_{j}$ from Fourier transform defined in any non-commutative algebra in dimension higher than two, not contradicting the fact that for $d=2$ the symmetric quaternionic Fourier transform [9, 3] does the job.

Theory of analytic functions, extended to the hypercomplex functions of hypercomplex variables, was first introduced in 1893 by G.W. Scheffers [35] and then extended by $[36,25,37]$. In particular, the existence of Cauchy formula in commutative hypercomplex algebras [38], implies existence of the corresponding Hilbert transform (5) in hypercomplex space.

Definition 2.7. The elliptic Scheffers algebra $\mathbb{S}_{d}$ is a unital, commutative and associative algebra over the field $\mathbb{R}$ of dimension $2^{d}$. It has generators $\left\{1, e_{1}, \ldots, e_{d}\right\}$ satisfying the conditions $e_{i}^{2}=-1, e_{i} e_{j}=e_{j} e_{i}, i, j=1, \ldots, d$. The basis of the algebra $\mathbb{S}_{d}$ consists of the elements of the form $e_{0}=1, e_{\beta}=e_{\beta_{1}} e_{\beta_{2}} \ldots e_{\beta_{s}}, \beta=\left(\beta_{1}, \ldots, \beta_{s}\right), \beta_{1}<\beta_{2}<$ $\cdots<\beta_{s}, 1 \leq s \leq d$. Each element $w \in \mathbb{S}_{d}$ has the form

$w=\sum_{\beta} w_{\beta} e_{\beta}=w_{0} e_{0}+\sum_{s=1}^{d} \sum_{\beta_{1}<\cdots<\beta_{s}} w_{\beta} e_{\beta}, \quad\left(w_{0}, w_{\beta}\right) \in \mathbb{R}$. 
Definition 2.8. The spaces $\mathbb{S}_{+}(i)$ and $\mathbb{S}_{+}^{d}, i=1, \ldots, d$, are defined by

$$
\begin{aligned}
& \mathbb{S}_{+}(i)=\left\{a+b e_{i} \mid a, b \in \mathbb{R} ; b>0\right\} \\
& \mathbb{S}_{+}^{d}=\bigoplus_{i=1}^{d} \mathbb{S}_{+}(i)
\end{aligned}
$$

Hypercomplex analytic signal is defined to be any holomorphic function $f: \mathbb{S}_{+}^{d} \rightarrow \mathbb{S}_{d}$ on the boundary $\partial \mathbb{S}_{+}^{d} \simeq \mathbb{R}^{d}$.

The Scheffers algebra valued Fourier transform of the function $f: \mathbb{R}^{d} \rightarrow \mathbb{R}$ is defined as follows

$$
\begin{aligned}
& \hat{f}(\boldsymbol{\omega})=\int_{-\infty}^{\infty} f\left(x_{1}, \ldots, x_{d}\right) \prod_{i=1}^{d} e^{-e_{i} \omega_{i} x_{i}} \mathrm{~d} x_{1} \ldots \mathrm{d} x_{d}, \\
& f(\boldsymbol{x})=\frac{1}{(2 \pi)^{d}} \int_{-\infty}^{\infty} f\left(\omega_{1}, \ldots, \omega_{d}\right) \prod_{i=1}^{d} e^{e_{i} \omega_{i} x_{i}} \mathrm{~d} \omega_{1} \ldots \mathrm{d} \omega_{d} .
\end{aligned}
$$

Theorem 2.9. The hypercomplex valued analytic signal $f_{h}$ : $\mathbb{R}^{d} \rightarrow \mathbb{S}_{d}$ of the real valued function $f: \mathbb{R}^{d} \rightarrow \mathbb{R}$ defined as

$$
\begin{aligned}
f_{h}(\boldsymbol{x}):= & \left\langle\hat{f}(\boldsymbol{\omega}), \alpha_{0 \ldots 0}(\boldsymbol{x}, \boldsymbol{\omega})\right\rangle_{+}(\boldsymbol{x}) \\
& +\sum_{i=1}^{d} e_{i}\left\langle\hat{f}(\boldsymbol{\omega}), \alpha_{0 \ldots 1(i) \ldots 0}(\boldsymbol{x}, \boldsymbol{\omega})\right\rangle_{+}(\boldsymbol{x}) \\
& +\sum_{i<j} e_{i} e_{j}\left\langle\hat{f}(\boldsymbol{\omega}), \alpha_{0 \ldots 1(i, j) \ldots 0}(\boldsymbol{x}, \boldsymbol{\omega})\right\rangle_{+}(\boldsymbol{x})+\ldots
\end{aligned}
$$

has as components the corresponding phase-shifted functions $f_{\boldsymbol{j}}(\boldsymbol{x})$, i.e.

$$
\begin{aligned}
f_{h}(\boldsymbol{x})=f(\boldsymbol{x}) & +\sum_{i=1}^{d} e_{i} f_{0 \ldots 1(i) \ldots 0}(\boldsymbol{x}) \\
& +\sum_{i<j} e_{i} e_{j} f_{0 \ldots 1(i, j) \ldots 0}(\boldsymbol{x})+\ldots
\end{aligned}
$$

Observation 2.10. As it is the case in 1-D, the hypercomplex Fourier transform of $f_{h}(\boldsymbol{x})$ is supported only over nonnegative frequencies, as we have:

$$
\hat{f}_{h}(\boldsymbol{\omega})=\prod_{i=1}^{d}\left(1+\operatorname{sign}\left(\omega_{i}\right)\right) \hat{f}\left(\omega_{1}, \ldots, \omega_{d}\right) .
$$

In practical situations, Bedrosian theorem [39] facilitates the computation of the Hilbert transform of product of functions with non-overlapping spectra. In [23] we extend this result to multidimensional analytic signals.

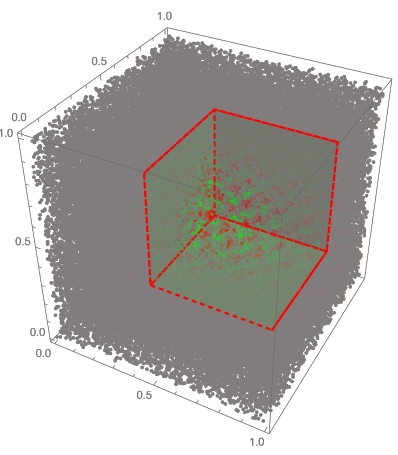

(a) Oscillating process

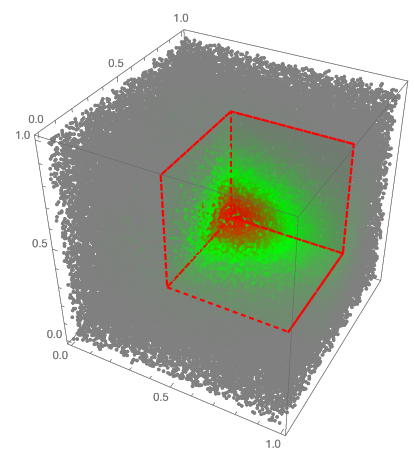

(b) Envelope
Fig. 1: Point cloud of cube with a deleted octant is shown. (a) Illustration of oscillating process $f(x, y, z)$ that takes place inside the cube; (b) Envelope function of the oscillating process.

\section{NUMERICAL EXAMPLES}

\subsection{Simple example in 3D}

As a direct illustration of how the construction of the envelope works, we provide an example in 3D. The oscillating function that we analyse is given by the cosine wave modulated by a gaussian window:

$$
\begin{aligned}
f(x, y, z) & =e^{-10 x^{2}-20 y^{2}-20 z^{2}} \cos (50 x) \cos (40 y) \cos (60 z) \\
& =f_{x}(x) f_{y}(y) f_{z}(z) .
\end{aligned}
$$

This particular toy example allows us to separate the variables when calculating the phase-shifted components $f_{\boldsymbol{j}}$ from (9). In three dimensions we will have in total $\left|\{0,1\}^{3}\right|=8$ shifted functions.

Calculation of some $f_{j}$ using (2) reduces to the calculation of forward and inverse cosine and sine transforms. For example for the $f_{100}$ shifted component, we will have

$$
\begin{aligned}
f_{100}(x, y, z) & =\frac{f_{y}(y) f_{z}(z)}{\pi} . \\
& \cdot \int_{0}^{\infty} \int_{-\infty}^{\infty} f_{x}(x) \sin \left(\omega_{1}\left(x-x^{\prime}\right)\right) \mathrm{d} x^{\prime} \mathrm{d} \omega_{1} .
\end{aligned}
$$

Taking into account that $f$ is even in each variable, after expanding the sine of difference, we see that we have to calculate only forward cosine and inverse sine transform. This double integral can be calculated into semi-analytical form by expressing it in terms of $\operatorname{erf}(z)=\int_{0}^{z} e^{-t^{2}} \mathrm{~d} t$. To illustrate the resulting envelope of signal (20), in Fig. 1 we vizualize a point cloud in a cube with a removed octant where each point is colour coded according to the signal value (the origin is shifted by 0.5 ). This method allows to visualize three dimensional signals over the inner faces of the deleted octant. In Fig. 1(a) the original $f(x, y, z)$ is plotted, while in Fig. 1(b) we plot the envelope function obtained from (3) computed on the 8 phase-shifted functions obtained similarly to (21). 


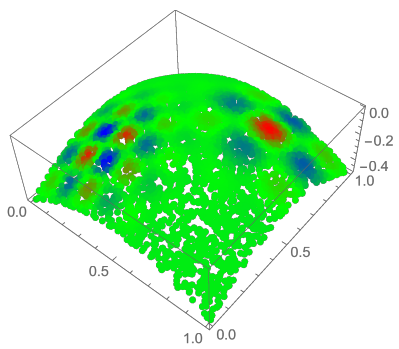

(a) signal over point cloud

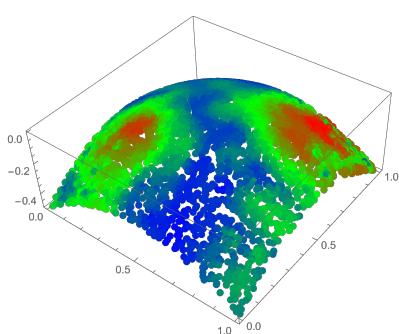

(c) envelope over point cloud

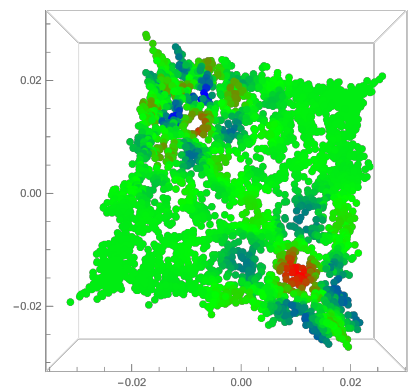

(b) signal in diffusion space

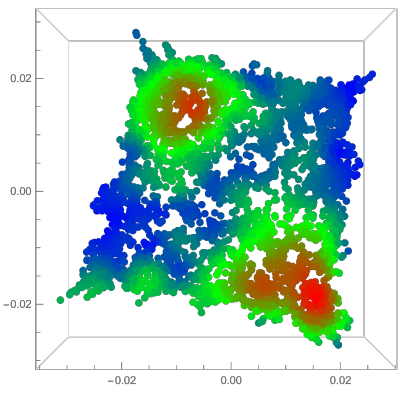

(d) envelope in diffusion space
Fig. 2: Point cloud and diffusion embedding of the corresponding knngraph. Oscillation process is given by the sum of modulated gaussians: (a) original point cloud with signal over it; (b) embedding of the point cloud by the first two eigenvectors of normalized Laplacian; (c) envelope of the original signal; (d) envelope of the original signal in diffusion space.

\subsection{Analytic signal on graph}

Our objective is to apply the multidimensional analytic signal method to study oscillating signals on graphs. Let us consider a graph $G=(V, E)$ consisting of the set of nodes $V$ and the set of edges $E$. The topology of a graph can be captured by a variety of operators like adjacency operator or Laplacian [40]. In machine learning scenario, data is frequently assumed to be noisy samples taken from some smooth manifold. Locally, the manifold is homeomorphic to some open ball in $\mathbb{R}^{d}$, so that we can easily apply the theory of multidimensional analytic signal on a patch of such a manifold. A now classical approach to embed a graph (or part of it) into $\mathbb{R}^{d}$, is to use the diffusion maps proposed by Coifman and Lafon [24].

Let us consider then the mapping $\phi: V \rightarrow \mathbb{R}^{d}$, for instance coming from the diffusion map [24]. Let us suppose also, that we have some oscillating process $f: V \rightarrow \mathbb{R}$ over the vertices of a graph. Our goal is to obtain some global information on this process, here instantaneous amplitude and phase. We illustrate the technique on a simple, yet edifying example. We consider a sum of two gaussians modulated by different frequencies that lie on a curved surface. The sampled patch is then given by a set of 4096 randomly picked points on the surface as displayed in Fig. 2(a). This resulting point cloud is inherently 2-dimensional although it is embedded in 3 dimensions. Then, to obtain an associated graph, we consider the $k=20$ nearest neighbours graph to mesh the point cloud. Using the diffusion map given by the first two eigenvectors of the normalised Laplacian, to embed the patch

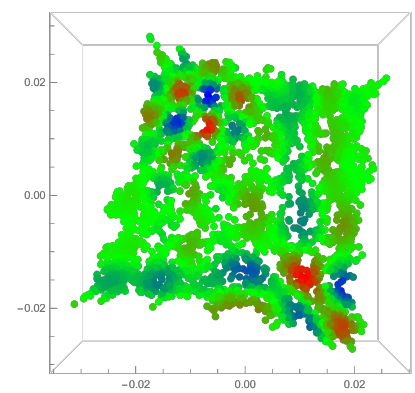

(a) $f_{00}$ shifted component

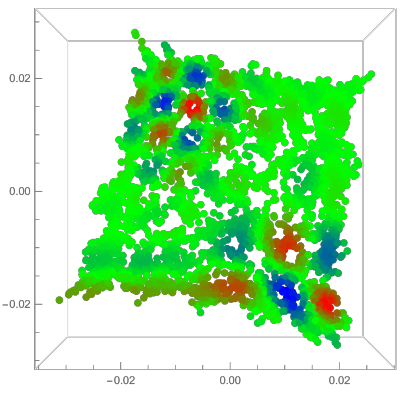

(a) $f_{01}$ shifted component

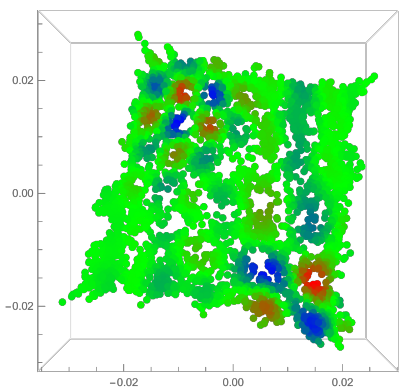

(b) $f_{10}$ shifted component

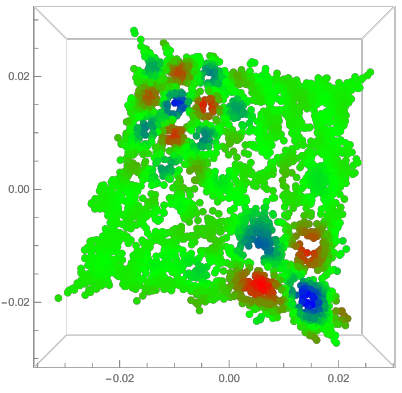

(b) $f_{11}$ shifted component
Fig. 3: Phase shifted components in diffusion space.

in a 2-D Euclidean space, the resulting embedding of the corresponding $k$-nn graph with the same oscillating process is shown in Fig. 2 (b).

To calculate the discrete version of hypercomplex Fourier transform $\hat{f}(\boldsymbol{\omega})$, we applied the transformation (14) to the discrete signal of Fig. 2(b). Then corresponding Scheffers algebra-valued analytic signal $f_{h}(\boldsymbol{x})$ was constructed, by first restricting the spectrum $\hat{f}(\boldsymbol{\omega})$ to positive frequencies according to (19) and applying inverse formula (15). All computations were performed in the diffusion map domain. The signal domain was rescaled by a factor of 10 for convenience. Discrete version of the Fourier transform (14) was calculated for the first 50 frequencies with step 1 . Then formula (19) was applied prior to the inverse discrete transform. In Fig. 2 (c) and (d), the envelope function of Eq. (3) is displayed on the original point cloud and in the diffusion space, respectively. Fig. 3 shows separate phase-shifted components $f_{\boldsymbol{j}}(\boldsymbol{x})$ for the four directions $\boldsymbol{j}=00,10,01$ and 11 in the diffusion space.

\section{CONCLUSION}

In this paper we developed basic theory for analytic signal in many dimensions and applied the discretized theory to signals supported on graphs. The presented hypercomplex analytic signal, aside being analytic function of hypercomplex variable, satisfies in many dimensions all the desirable properties that we expect from 1-D analytic signals. Future work will show how the presented theory will be used in the applications from multidimensional data analysis and machine learning for global characterization of locally oscillating processes. 


\section{REFERENCES}

[1] D. Gabor, "Theory of communication," Journal of the Institution of Electrical Engineers-Part III: Radio and Communication Engineering, vol. 93, no. 26, pp. 429-441, 1946.

[2] D. Vakman, "On the analytic signal, the Teager-Kaiser energy algorithm, and other methods for defining amplitude and frequency," IEEE Transactions on Signal Processing, vol. 44, no. 4, pp. 791-797, 1996.

[3] T. A. Ell, N. Le Bihan, and S. J. Sangwine, Quaternion Fourier transforms for signal and image processing, John Wiley \& Sons, 2014.

[4] T. A. Ell, "Hypercomplex spectral transformations, PhD Dissertation," 1992.

[5] T. A. Ell and S. J. Sangwine, "Hypercomplex Fourier transforms of color images," IEEE Transactions on image processing, vol. 16, no. 1, pp. 22-35, 2007.

[6] M. Felsberg and G. Sommer, "The monogenic signal," IEEE Transactions on Signal Processing, vol. 49, no. 12, pp. 3136-3144, 2001.

[7] D. Alfsmann, H. G. Göckler, S. J. Sangwine, and T. A. Ell, "Hypercomplex algebras in digital signal processing: Benefits and drawbacks," in Signal Processing Conference, 2007 15th European. IEEE, 2007, pp. 1322-1326.

[8] S. L. Hahn and K. M. Snopek, Complex and Hypercomplex Analytic Signals: Theory and Applications, Artech House, 2016.

[9] T. Bülow and G. Sommer, "Hypercomplex signals-a novel extension of the analytic signal to the multidimensional case," IEEE Transactions on signal processing, vol. 49, no. 11, pp. 2844-2852, 2001.

[10] S. J. Sangwine and N. Le Bihan, "Hypercomplex analytic signals: extension of the analytic signal concept to complex signals," in Signal Processing Conference, 2007 15th European. IEEE, 2007, pp. 621624.

[11] S. Hahn and K. Snopek, "Quasi-analytic multidimensional signals," Bulletin of the Polish Academy of Sciences: Technical Sciences, vol. 61, no. 4, pp. 1017-1024, 2013.

[12] N. Le Bihan and S. J. Sangwine, "The hyperanalytic signal," arXiv preprint arXiv:1006.4751, 2010.

[13] S. Bernstein, "The fractional monogenic signal," in Hypercomplex Analysis: New Perspectives and Applications, pp. 75-88. Springer, 2014.

[14] C. P. Bridge, "Introduction to the monogenic signal," arXiv preprint arXiv:1703.09199, 2017.

[15] H. Zhang, "Multidimensional analytic signals and the Bedrosian identity," Integral Equations and Operator Theory, vol. 78, no. 3, pp. 301321, 2014.

[16] R. R. Coifman, S. Lafon, A. B. Lee, M. Maggioni, B. Nadler, F. Warner, and S. W. Zucker, "Geometric diffusions as a tool for harmonic analysis and structure definition of data: Diffusion maps," Proceedings of the National Academy of Sciences of the United States of America, vol. 102, no. 21, pp. 7426-7431, 2005.

[17] S. T. Roweis and L. K. Saul, "Nonlinear dimensionality reduction by locally linear embedding," science, vol. 290, no. 5500, pp. 2323-2326, 2000.

[18] D. I. Shuman, S. K. Narang, P. Frossard, A. Ortega, and P. Vandergheynst, "The emerging field of signal processing on graphs: Extending high-dimensional data analysis to networks and other irregular domains," IEEE Signal Processing Magazine, vol. 30, no. 3, pp. 83-98, 2013.

[19] M. Aubry, U. Schlickewei, and D. Cremers, "The wave kernel signature: A quantum mechanical approach to shape analysis," in Computer Vision Workshops (ICCV Workshops), 2011 IEEE International Conference on. IEEE, 2011, pp. 1626-1633.
[20] I. Kokkinos, M. M. Bronstein, R. Litman, and A. M. Bronstein, "Intrinsic shape context descriptors for deformable shapes," in Computer Vision and Pattern Recognition (CVPR), 2012 IEEE Conference on. IEEE, 2012, pp. 159-166.

[21] A. Venkitaraman, S. Chatterjee, and P. Handel, "On Hilbert transform of signals on graphs," Proc. Sampling Theory Appl, 2015.

[22] A. Venkitaraman, S. Chatterjee, and P. Händel, "Hilbert transform, analytic signal, and modulation analysis for graph signal processing," arXiv preprint arXiv:1611.05269, 2016.

[23] M. Tsitsvero, P. Borgnat, and P. Gonçalves, "Analytic signal in many dimensions," arXiv preprint arXiv:1712.09350, 2017.

[24] R. R. Coifman and S. Lafon, "Diffusion maps," Applied and computational harmonic analysis, vol. 21, no. 1, pp. 5-30, 2006.

[25] V. S. Vladimirov and I. V. Volovich, "Superanalysis. ii. Integral calculus," Theoretical and Mathematical Physics, vol. 60, no. 2, pp. 743765, 1984.

[26] F. Brackx, N. De Schepper, and F. Sommen, "The two-dimensional Clifford-Fourier transform," Journal of mathematical Imaging and $\mathrm{Vi}$ sion, vol. 26, no. 1-2, pp. 5-18, 2006.

[27] S. Hahn and K. Snopek, "The unified theory of n-dimensional complex and hypercomplex analytic signals," Bulletin of the Polish Academy of Sciences: Technical Sciences, vol. 59, no. 2, pp. 167-181, 2011.

[28] N. Le Bihan, S. J. Sangwine, and T. A. Ell, "Instantaneous frequency and amplitude of orthocomplex modulated signals based on quaternion Fourier transform," Signal Processing, vol. 94, pp. 308-318, 2014.

[29] N. Le Bihan and S. Sangwine, "The H-analytic signal," in 16th European Signal Processing Conference (EUSIPCO-2008), 2008, pp. P8-1.

[30] S. Bernstein, J.-L. Bouchot, M. Reinhardt, and B. Heise, "Generalized analytic signals in image processing: comparison, theory and applications," in Quaternion and Clifford Fourier Transforms and Wavelets, pp. 221-246. Springer, 2013.

[31] H. De Bie, "Clifford algebras, Fourier transforms, and quantum mechanics," Mathematical Methods in the Applied Sciences, vol. 35, no. 18, pp. 2198-2228, 2012.

[32] H. De Bie, N. De Schepper, and F. Sommen, "The class of CliffordFourier transforms," Journal of Fourier Analysis and Applications, vol. 17, no. 6, pp. 1198-1231, 2011.

[33] M. Felsberg, T. Bülow, G. Sommer, and V. M. Chernov, "Fast algorithms of hypercomplex Fourier transforms," in Geometric computing with Clifford algebras, pp. 231-254. Springer, 2001.

[34] P. Singh and S. D. Joshi, "Some studies on multidimensional Fourier theory for Hilbert transform, analytic signal and space-time series analysis," arXiv preprint arXiv:1507.08117, 2015.

[35] M. Scheffers, "Sur la généralisation des fonctions analytiques," $C R$ Acad. Sc, vol. 116, pp. 1114, 1893.

[36] P. Ketchum, "Analytic functions of hypercomplex variables," Transactions of the American Mathematical Society, vol. 30, no. 4, pp. 641$667,1928$.

[37] F. A. Berezin, Introduction to superanalysis, vol. 9, Springer Science \& Business Media, 2013.

[38] P. S. Pedersen, "Cauchy's integral theorem on a finitely generated, real, commutative, and associative algebra," Advances in mathematics, vol. 131, no. 2, pp. 344-356, 1997.

[39] E. Bedrosian, "A product theorem for Hilbert transforms," Technical report, 1962.

[40] F. R. Chung, Spectral graph theory, American Mathematical Soc., 1997. 